\title{
Unusual amplification in polymerase chain reaction for a plasmid containing an insert derived from bovine genomic DNA
}

\author{
Satoko Isemura ${ }^{1}$, Akane Imai $^{2}$, Eiichi Saitoh $^{3}$, Mamoru Isemura $^{4^{*}}$ \\ ${ }^{1}$ Department of Dental Hygiene, The Nippon Dental University College at Niigata, Niigata, Japan \\ ${ }^{2}$ Department of Biochemistry, School of Life Dentistry at Niigata, The Nippon Dental University, Niigata, Japan \\ ${ }^{3}$ Graduate School of Technology, Niigata Institute of Technology, Niigata, Japan \\ ${ }^{4}$ Graduate School of Food and Nutritional Sciences, University of Shizuoka, Shizuoka, Japan; \\ *Corresponding Author: isemura@u-shizuoka-ken.ac.jp
}

Received 28 April 2012; revised 24 May 2012; accepted 14 June 2012

\section{ABSTRACT}

The saliva of various animals contains prolinerich proteins which may play important roles in prevention of mineral precipitation, protection of dietary and digestive proteins from interaction with tannins, and modulation of bacterial colonization on the tooth surface. Previously, we found a segment of Escherichia coli genomic DNA in bovine tooth germ mRNA encoding the prolinerich protein $\mathrm{P}-\mathrm{B}$. To examine whether $E$. coli genomic DNA is present in bovine genomic DNA, we constructed a plasmid library from the bovine DNA. Although results so far have failed to indicate any such presence in the bovine nucleotides examined, experiments using the polymerase chain reaction (PCR) revealed unusual amplification of nucleotides. As an initial step of the study on possible occurrence of $E$. coli-derived nucleotide sequence in bovine genomic DNA of P-B, we examined the structure of the PCR products generated by unexpected amplification. The determined structure of the PCR products suggested that when the two single strand chains that grow by reading the sequence of the respective template reached a hybridizable short nucleotide structure, they became hybridized and subsequent elongation was continued by reading the sequence of the counter chain that had been elongated by reading the template. It is possible that elongation of the chain was interrupted once before the completion of amplification due to the template's palindrome region which had formed a double strand structure during the PCR process. Such an unusual amplification made possible under certain conditions in a DNA sequence may be one of the mechanisms for the genetic recombination found in our previous study.

Keywords: Bovine Genomic DNA; PCR Amplification; Nucleotide Sequence; Palindrome

\section{INTRODUCTION}

The saliva of various animals contains proline-rich proteins which may play important roles in prevention of mineral precipitation, protection of dietary and digestive proteins from interaction with tannins, and modulation of bacterial colonization on the tooth surface [1-4]. We have isolated a proline-rich protein termed P-B from human saliva and determined its amino acid and nucleotide sequences [5]. Following the finding of Strawich and Glimcher that a homologous protein is present in bovine tooth germ [6], we started a study on bovine P-B [7]. In the course of cloning bovine cDNA for P-B, we found a segment of E. coli genomic DNA in bovine tooth germ mRNA encoding P-B [8]. Consequently, we examined the possible occurrence of E. coli DNA-derived nucleotide sequences in bovine genomic DNA using a plasmid library constructed from EcoRV digests of the bovine DNA. Results so far have failed to indicate the presence of $E$. coli DNA in the bovine nucleotides examined, but they revealed unusual amplification by the polymerase chain reaction (PCR). We report here findings to suggest that a specific nucleotide sequence in combination with the palindrome structure can promote preferential hybridization between a short nucleotide and its complementary structure resulting in the generation of unexpected PCR products.

\section{MATERIALS AND METHODS}




\subsection{Materials}

The LA PCR kit, agarose (H14 TAKARA), restriction enzymes, and SYBR Green I Nucleic Acid Gel Stain were purchased from Takara Bio Inc. (Otsu, Japan); SynerGel for use with agarose, from Diversified Biotech Inc. (Boston, MA, USA); Bovine genomic DNA and Perfectly Blunt Cloning Kits with the plasmid pT7Blue vector, from Novagen, Inc. (Madison, WI, USA); the GenElute Five-minutes Plasmid Miniprep Kit and DNA standard for gel electrophoresis, from Sigma-Aldrich Japan, Inc. (Tokyo, Japan); and the Big Dye Terminator cycle sequencing kit, Applied Biosystems Japan Ltd. (Tokyo, Japan).

Primers used were as follows:

M13-47: 5'-CGCCAGGGTTTTCCCAGTCACGAC3' RV-M: 5'-GAGCGGATAACAATTTCACACAGG-3' T7: 5'-TAATACGACTCACTATAGGG-3' pT7Blue9: 5'GATTACGCCAAGCTCTAATA-3' HindR: 5'-AAGCTTGCATGCCTGCAGGT-3' B6C2985: 5'-ACCCGGGGATCCGATATCTT-3'

M13-47, RV-M, and T7 Bos Best ${ }^{\mathrm{TM}}$ sequencing primers were obtained from Takara Bio. Inc. The others were custom-made products of Invitrogen Life Technologies (Tokyo, Japan). Their positions in the plasmid B6 are indicated in Figure 1 and/or Figure 6(b).

\subsection{Agarose Gel Electrophoresis}

Agarose gel (1\%) was used for the electrophoresis of nucleotide sequences longer than $1000 \mathrm{bp}$. For shorter sequences, gels prepared from $0.7 \%$ agarose and $1.2 \%$ Syner gel according to the manufacturer's instructions were used. Nucleotides were visualized by staining with SYBR Green I.

\subsection{Plasmid Library}

Bovine genomic DNA (50 $\mu \mathrm{g})$ was digested with EcoRV (450 U) in $250 \mu$ l of universal buffer H (Takara) and fractionated by agarose gel electrophoresis. Using a gel section containing 3000 - $4200 \mathrm{bp}$ and the vector pT7Blue, we constructed a clone-pool library in 96-well plates. From the agar culture plate containing E. coli with plasmids from each well, 10 colonies each were removed from wells A1-H12 to prepare plasmid mixtures termed a1-h12, respectively.

\subsection{PCR}

PCR was performed using a long and accurate (LA) PCR kit suitable for amplification of long DNA according to the manufacturer's instructions. Plasmid mixtures, cloned plasmids or restriction enzyme digests were subjected to PCR amplification (40 cycles of denaturation at $95^{\circ} \mathrm{C}$ for $30 \mathrm{sec}$, annealing at $55^{\circ} \mathrm{C}$ for $30 \mathrm{sec}$, and elon- gation at $72^{\circ} \mathrm{C}$ for $\left.1 \mathrm{~min}\right)$ in a total volume of $10 \mu \mathrm{l}(1 \mu \mathrm{l}$ each of forward and reverse primers $(2 \mu \mathrm{M}), 10 \mathrm{ng}$ of template, $5 \mu$ of One Shot LAPCR Mix, and water to give a final volume of $10 \mu \mathrm{l}$ ) using MyCycler Thermal Cycler (BIO-RAD, Hercules, CA, USA).

\subsection{Restriction Enzyme Digestion}

Plasmids were digested with HindIII or EcoRI at $37^{\circ} \mathrm{C}$ overnight according to the manufacturer's instructions.

\subsection{Cloning of $\mathrm{B} 6$}

B6 was cloned from well G12 according to methods described previously $[5,8]$.

\subsection{Nucleotide Sequence}

Nucleotide sequences were analyzed by the dye terminator method on an ABI PRISM 310NT Genetic Analyzer (Applied Biosystems Japan Ltd., Tokyo, Japan) as described previously $[5,8]$.

\section{RESULTS}

In this study, we prepared 96 plasmid mixtures (a1h12) expected to contain $3000-4200 \mathrm{bp}$ inserts derived from bovine genomic DNA from 10 colonies of $E$. coli with the vector pT7Blue. The plasmid mixture derived from well G12 was named g12. According to the assumed plasmid structure shown in Figure 1, we expected PCR using the primers M13-47 and RV-M to give a nucleotide product larger than $3000 \mathrm{bp}$. However, the major product was much smaller (ca. $300 \mathrm{bp}$ ) as shown in

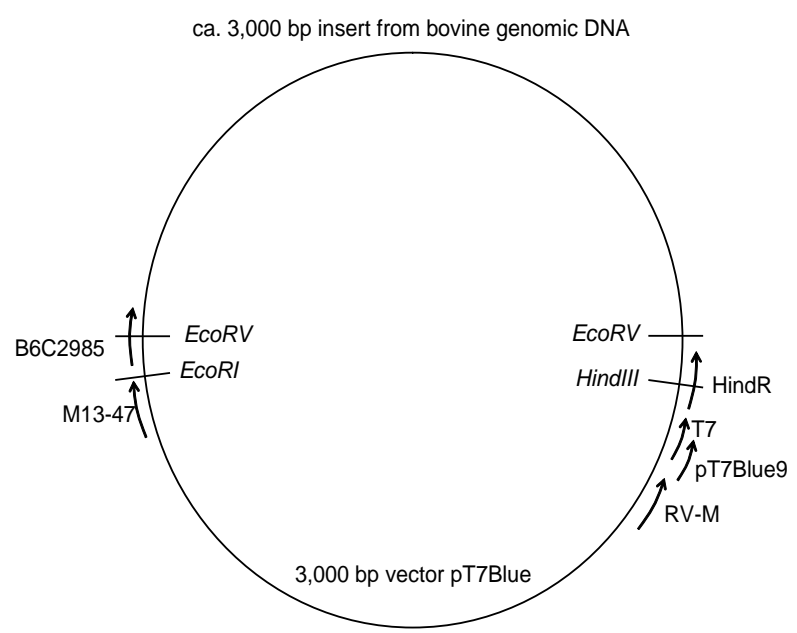

Figure 1. A schematic overview of the assumed structure of a constructed plasmid, g12. A plasmid library was prepared from $E c o R V$ digests (ca. $3000 \mathrm{bp}$ ) of bovine genomic DNA ligated with the vector pT7Blue. The plasmid mixture g12 was selected from 10 colonies of $E$. coli. Restriction enzyme sites are shown. The positions of the sequence used for designing primers are indicated by arrows. 
Figure 2(a). If the RV-M or M13-47 sequence is present in the insert near the EcoRV site, PCR using RV-M and M13-47 might give a product of such size, but PCR with the HindIII digest of g12 would not (see Figure 1). When PCR was carried out using the HindIII digest of g12, the product was ca. 150 bp (Figure 2(b)), suggesting it to be unlikely that the RV-M or M13-47 sequence is present in the insert.

The nucleotide sequence of ca. $150 \mathrm{bp}$ termed pn-150 thus obtained was determined as shown in Figure 3(a). This finding indicated that pn-150 contained the 19-base sequence TTAAAGCAGTAGCGTATTG, between the EcoRV site extending from the M13-47 sequence in the vector and the vector sequence extending from RV-M, and that this portion was not derived from pT7Blue (Figure 3(a)). Therefore, this section was considered to be derived from bovine genomic DNA.

To explain this unexpected result, we cloned the plasmid which gave a 300-bp PCR product using g12, and obtained a plasmid termed B6. When we digested B6 with HindIII on the basis that pT7Blue contains a single HindIII site, we obtained a band of ca. 6000 bp (Figure 4(a)), indicating that B6 was of the expected size. PCR using the intact B6 and a combination of forward (M13$47)$ and reverse $(\mathrm{RV}-\mathrm{M})$ primers gave a major product of ca. 300 bp termed b6-300 (Figure 4(b)). Thus, the plasmid B6 gave a 300-bp PCR product in the plasmid mixture g12. B6 gave products of less than $300 \mathrm{bp}$ when we used primers to bind inner positions directed at the insert instead of the combination of M13-47 and RV-M (Figure 5, see also Figures 1 and 6(b)), indicating that amplifycation similar to that with primers of M13-47 and RV-M occurred using other primers under the present conditions.

A portion of bovine genomic DNA in B6 was sequenced and registered as "Bos taurus DNA, palindrome sequence region" in NCBI GenBank (AB511281) (see Figure 6(a)). The nucleotide sequence of $\mathrm{B} 6$ around the EcoRV site together with $124 \mathrm{bp}$ from RV-M and $71 \mathrm{bp}$ from the M13-47 side is shown in Figure 6(b). The sites for which primers were designed are also indicated (see also Figure 1).

Based on the structure of B6, we explain how the HindIII digest of g12 gave the PCR product shown in Figure 3(a) (Figure 3(b)). When the nucleotide chain elongated from RV-M reached $\mathrm{C}^{-87}-\mathrm{A}-\mathrm{T}^{-85}$ (numbering based on the sequence shown in Figure 6(b)), this portion hybridized with $\mathrm{G}^{2946(-)}-\mathrm{T}-\mathrm{A}^{2948(-)}$ of the chain which had been elongated from the 5'-end of M13-47, and vice versa (Figure 3(b)). Subsequent elongation of each chain proceeded by reading the sequence of the counter chain as shown in Figure 3(b) to give pn-150.

When the primer M13-47 or RV-M was used to determine the nucleotide sequence of b6-300 (see Figure 4(b)), we were unsuccessful for unknown reasons. When T7 was used for sequencing, a partial sequence of b6-300

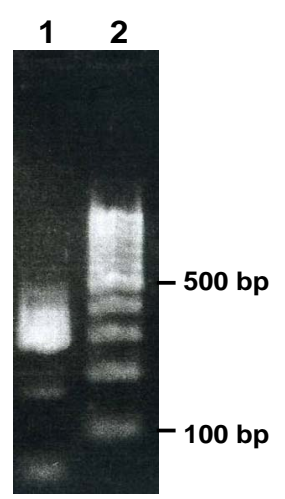

(a)

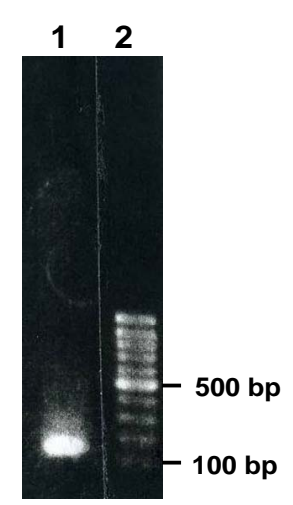

(b)
Figure 2. (a) Gel electrophoresis of the products of PCR using g12 as a template and the primers M13-47 and RV-M. Products of PCR (lane 1) and a size marker with multiples of 100 bp (lane 2); (b) Nucleotide (pn-150) produced by PCR of the HindIII digest of g12 (lane 1) and the size marker (lane 2).

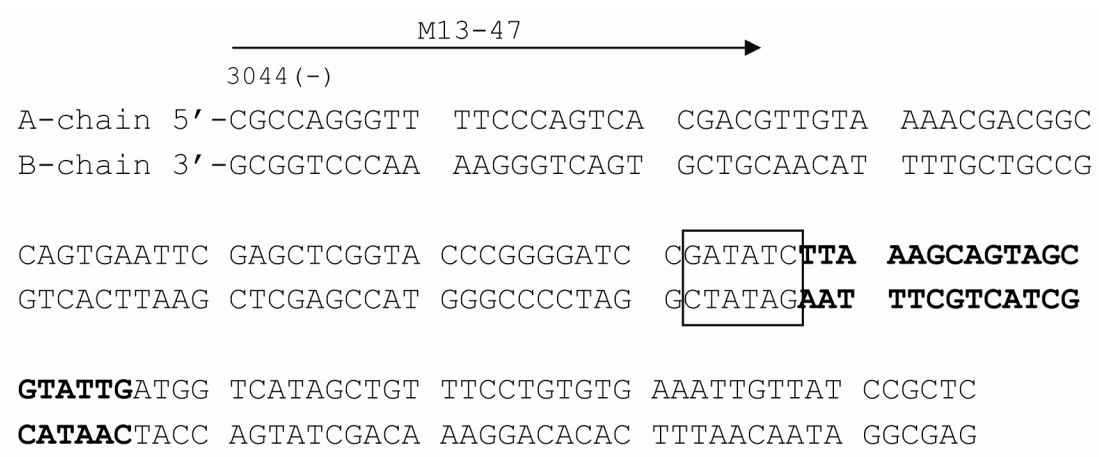

$-124$

RV-M

(a) 


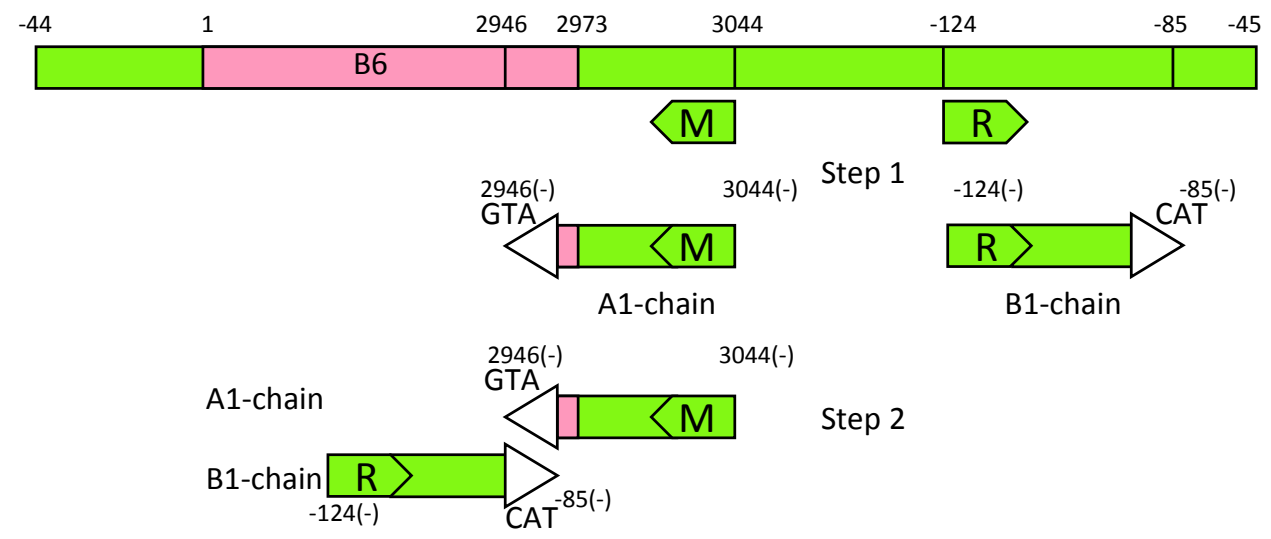

Step 3

PCR product (pn-150)

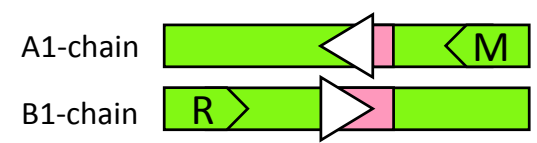

(b)

Figure 3. (a) Nucleotide sequence of pn-150. Arrows indicate primer sequences. The bold-face nucleotides show the structure presumably derived from bovine genomic DNA. The EcoRV site is boxed. Base numbers are based on those of the structure shown in Figure 6(b); (b) A possible mechanism for PCR amplification of pn-150. After the M13-47 primer (M) bound to the complintary sequence starting at $\mathrm{G}^{3044}$ in the template, elongation continued to make A1-chain with a terminal $-\mathrm{A}-\mathrm{T}-\mathrm{G}^{2946(-)}$ structure. B1-chain was produced by initial binding of RV-M (R) to the template structure starting at $\mathrm{G}^{-124}$, followed by elongation ill the completion of the trinucleotide structure of $-\mathrm{C}-\mathrm{A}-\mathrm{T}^{-85(-)}$ (Step 1). After hybridization between $\mathrm{G}^{2946(-)}$-T-A and C-A-T ${ }^{-85}$ (Step 2), A1-chain was elongated according to the sequence of $\mathrm{B} 1$ chain to complete A-chain and vice versa (Step 3), resulting in the formation of pn-150. The pink-colored segment represents the seence contained in B6 and green-colored sequences are those associated with the vector.

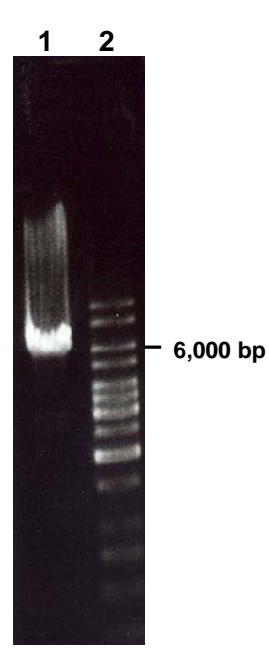

(a)

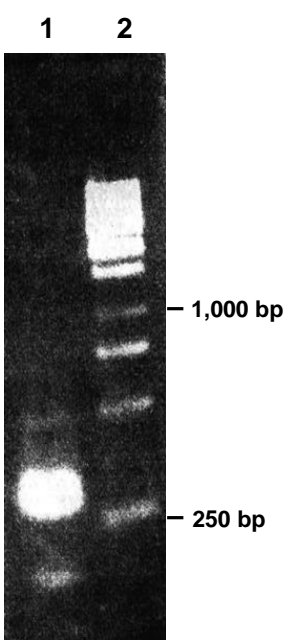

(b)

Figure 4. (a) Agarose-gel electrophoresis of the HindIII digest of B6 (lane 1) and a size marker (lane 2); (b) Agarose-gel electrooresis of the nucleotide (b6-300) produced by PCR using the HindIII digest of B6 and primers M13-47 and RV-M (lane 1) and a size marker (lane 2).

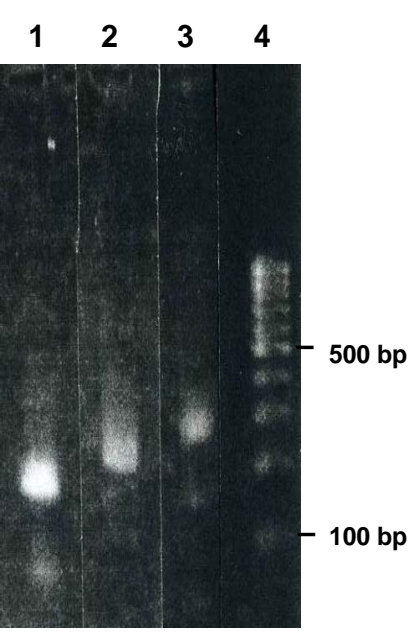

Figure 5. Agarose-gel electrophoresis of the nucleotides produced by PCR using B6 and the primers M13-47 and HindR (lane 1), B6 and the primers M13-47 and pT7Blue9 (lane 2), B6 and the primers M13-47 and RV-M (lane 3), and a size marker (lane 4). 
1 GATATCTTAA AgCAGTAGCG TATTGATGAT GAagtgagtG AGAGGCTCTG GCCTCCTCAA 61 TCACAGTAGC CACTGTATTT CTGGTTAACA AGTTAGCCAA ATCATTAAAG GCATTTTAAG 121 GGCCGGGCAA TCTGGAATGA GCCCAAATGT GTCCAATGAA AAATACTTAA TTTCTTTTCC 181 AAATTTAGTT AACAAATTAA ATATGGTAGT TTTATTTTCA GGCAAAACCG CAGTTTCAAT 241 GTGTGGAAAC AACCTGACAA TATACTGAGA ATCAGAATAA AGGTTAAATT CCTCATCTGC 301 AAACATAGCA AAAGCCTCTA TGACTGCTGT TATTTCAGCT CTTTGAGCTG AAGTTTCCCG 361 TGTTTCTAAA ACCTTTTGGT AATTTTTAGT AACTATGGAG GCTTTACCAT TTGCTGACCC 421 GTCAGTAAAG GCTATCTGAG CATTTGGAAT GGAGATTGTT TACATCTGAC AGGAAAATA 481 ACTGGATGTC TGGATATAAA ATTCAGCAAA ACATGTGAGG GTAAATGATG CAAAATTTTG 541 ACCAAAATAG TTTCCTATAG CAATCTGCCA AATTTTATCA AACATTAGAA GAGCAACAAG 601 TTGTTCCTTA TTATATGGAA TTACAATTTC TGATGGCTCT TTACCAAATA ATTCAATGTT 661 CCGCTTTTTT CCTTTAATAT CAAAGTAGCT ATCAATCCCG GATAAGAAGC CACTACATTT 721 TTAGTTTGAG CGGGAAGATG GACCCACTCT AGGGGGCCGT TTTTTTTATA AAACCAATTT 781 TTTGTCTGGT TATCCCAGTT ACACCTATGG GGGGGTTTTA TGGCAAAGGA ATTGTCAAGC 841 AGTTGTCAAT TTAATTTTTT AAAATTTTTT TAAAAGCCTT TATTTTTTTT AAAGCCTTTA 901 TTTGATTGCT TAAAAATTGA TCCTAATTCC AGTTCTAAGA GAAAGTTAAC TAATGAGACA 961 GAGTTAGCTC TTGTTGAAGT GTATGAGACT TTAAATGATC AGTTAATTAG GATTAATACT 1021 ACCAAAAGAT GAGATTAAAT TATTCTTGCC ATAAAACATA CACCTACAGG GTGATTGTGA 1081 CAAAAAGGCC CATTGGTTCC ATCTACCAGT GATCCCAAGA AAAATAGTTT TATCTTATCC 1141 CAGCTTGGTG GCACAATTAA TTATAAAACG AGgAAAAAG AAGTGTGGAG CTTTTTAGAA 1201 AAGAAGTAGC AAATATAATA ATTCCATTCA ATAAGGATCA ACTACAAGTC TTTTACAATA 1261 TAGTGATTGG CAAGTTGCCC TGATAGATTT CAGGGGTCAA ATTTTGTTTC ATTTGCCCTC 1321 AAGCCCCGAC AGTCGTTAAA AGTTCAAAAA TGTTAGATTG GCAGTTTGAG GATACCTGTG 1381 GAACTTTCCA ACTGTATGTA GTTCCTACGC TCCCCTTTAC CCTATGGGGG AGGGACGTGC 1441 TGTCTCAAAT AGGAGATACT CAGAGAAGGG TTTTCTCAAT TTCTTAGTTA TCAACAGGTG 1501 GTACAATTAA CAATACTTTA TATATTGTTA TAAATACGTA ATATAAATAT ATTTGCCTAA 1561 CTACAGTTTG CCTAATTAGG TATGGGTATA AATAAAACAT AATAAAGGTA TACCTAATTC 1621 TATTTATAGA TCTATACTTA CTTAATTTGT ATATAAATCA ATATATGTAT TATATATATA 1681 CTGTATAATT AAATGTATAT TGCAGTAATA TAATGTGTGT GGCTGATATT AATTGGTATG 1741 GATTGATGTG CTGTGATATA TGTTCTGTAT GCTGTATAAT TATATATGTG TGACATGTAT 1801 TATTACAGTA CATTGGTTTG TGTTGGTTGG TATTGGCTGT GTGCGTGTTG TATTGCTGTA 1861 ATATATATGA ATTAATATAT TAATTATAAG ATTAATTCAA GTATATTGAT TTATATATAT 1921 TGTATGTCAA TAAGTTTATA CTTGTTGCCA TATATAAATA CATTTTGCAT TTAGGTATAT 1981 CTGTATACTA AAATGAGATA AGGAGGTTAT ACATTTATTA TTTAAATTTA TACGGAGACC 2041 TAAACTAAAC ATTAGACCTA AATTAACATA TCTCTAAATT TATGTTGTTA AAATGTAAAT 2101 TTTAAAAAAT TTTAAAAAAT TAAATTGACA ACTGCTTGAC AATTCCTTTG CCATAAAACC 2161 CCCCCATAGG TGTAACTGGG ATAACCAGAC AAAAAATTGG TTTTATAAAA AAAACGGCCC 2221 CCTAGAGTGG GTCCATCTTC CCGCTCAAAC TAAAAATGTA GTGGCTTCTT ATCCGGGATT 2281 GATAGCTACT TTGATATTAA AGGAAAAAAG CGGAACATTG AATTATTTGG TAAAGAGCCA 2341 TCAGAAATTG TAATTCCATA TAATAAGGA CAACTTGTTG CTCTTCTAAT GTTTGATAAA 2401 ATTTGGCAGA TTGCTATAGG AAACTATTTT GGTCAAAATT TTGCATCATT TACCCTCACA 2461 TGTTTTGCTG AATTTTATAT CCAGACATCC AGTTATTTTT CCTGTCAGAT GTAAACAATC 2521 TCCATTCCAA ATGCTCAGAT AGCCTTTACT GACGGGTCAG CAAATGGTAA AGCCTCCATA 2581 GTTACTAAAA ATTACCAAAA GGTTTTAGAA ACACGgGAAA CTTCAGCTCA AAGAGCTGAA 2641 ATAACAGCAG TCATAGAGGC TTTTGCTATG TTTGCAGATG AGGAATTTAA CCTTTATTCT 2701 GATTCTCAGT ATATTGTCAG GTTGTTTCCA CACATTGAAA CTGCGGTTTT GCCTGAAAT 2761 AAAACTACCA TATTTAATTT GTTAACTAAA TTTGGAAAAG AAATTAAGTA TTTTTCATTG 2821 GACACATTTG GGCTCATTCC AGATTGCCCG GCCCTTAAAA TGCCTTTAAT GATTTGGCTA 2881 ACTTGTTAAC CAGAAATACA GTGGCTACTG TGATTGAGGA GGCCAGAGCC TCTCACTCAC 2941 TTCATCATCA ATACGCTACT GCTTTAAGAT ATC 2973 


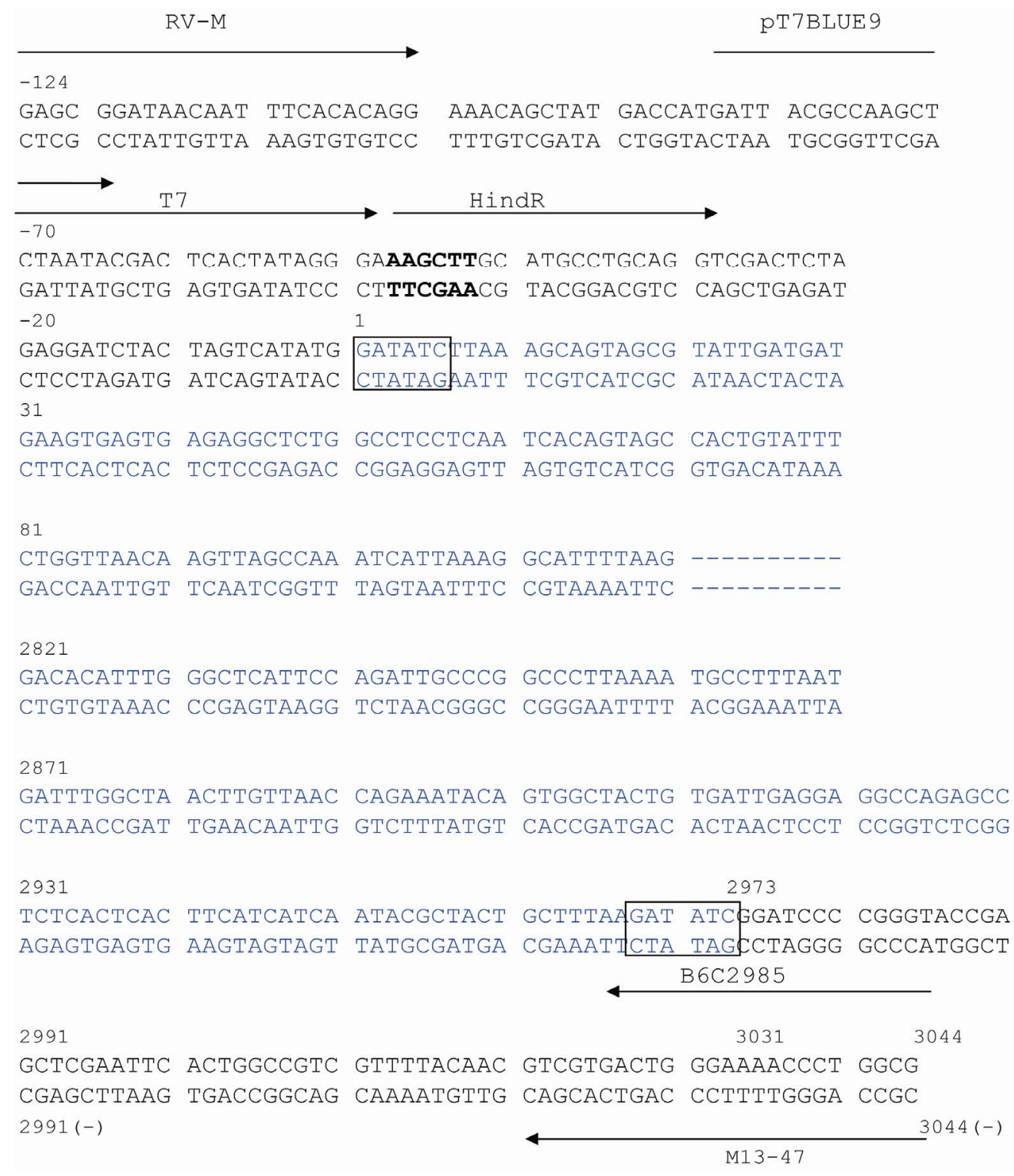

(b)

Figure 6. (a) Single chain of the nucleotide sequence of the cloned plasmid B6 containing sequences derived from bovine genomic DNA and the vector pT7Blue. EcoRV sites are indicated in bold face. The sequence of $\mathrm{G}^{1}-\mathrm{T}^{870}$ is the same as the complementary structure of $\mathrm{C}^{2973}-\mathrm{A}^{2104}$; (b) Nucleotide sequence of B6 around the EcoRV site (boxed) used as the cloning site. Numbering was started at $\mathrm{G}^{1}$ of the EcoRV site. The 5'-terminal was assigned as -124 and the 3 '-terminal as 3044 . Numbering for the counterchain was presented by addition of a mark (-) after a number as exemplified by 3044(-) for its 5'-terminal. The sequences used as primers are indicated by arrows. The HindIII site is indicated in bold face. The blue-colored sequence represents B6.

was determined as shown in Figure 7(a). The finding indicated that after a portion of $\mathrm{T}^{61}-\mathrm{C}-\mathrm{A}-\mathrm{C}^{64}$ in the growing chain hybridized to $\mathrm{A}^{37}-\mathrm{G}-\mathrm{T}-\mathrm{G}^{34}$ in the growing counter chain, the extension continued in the 3 'direction by reading the sequence of the counter chain preformed by PCR (Figure 7(b)). The finding suggests that two newly synthesized chains which had started from the primer $\mathrm{T} 7$ were hybridized to give a double strand polynucleotide shown in Figure 7(a), which represents an inner portion of the polynucleotide of b6-300.

When PCR was performed using the EcoRI digest of B6 as the template and primers B6C2985 and M13-47, a product of ca. 110 nucleotides was amplified and termed b6-110 (Figure 8(a)). The nucleotide b6-110 was sequenced as shown in Figure 8(b). It is suggested that preferential hybridization occurred between the 5-base 


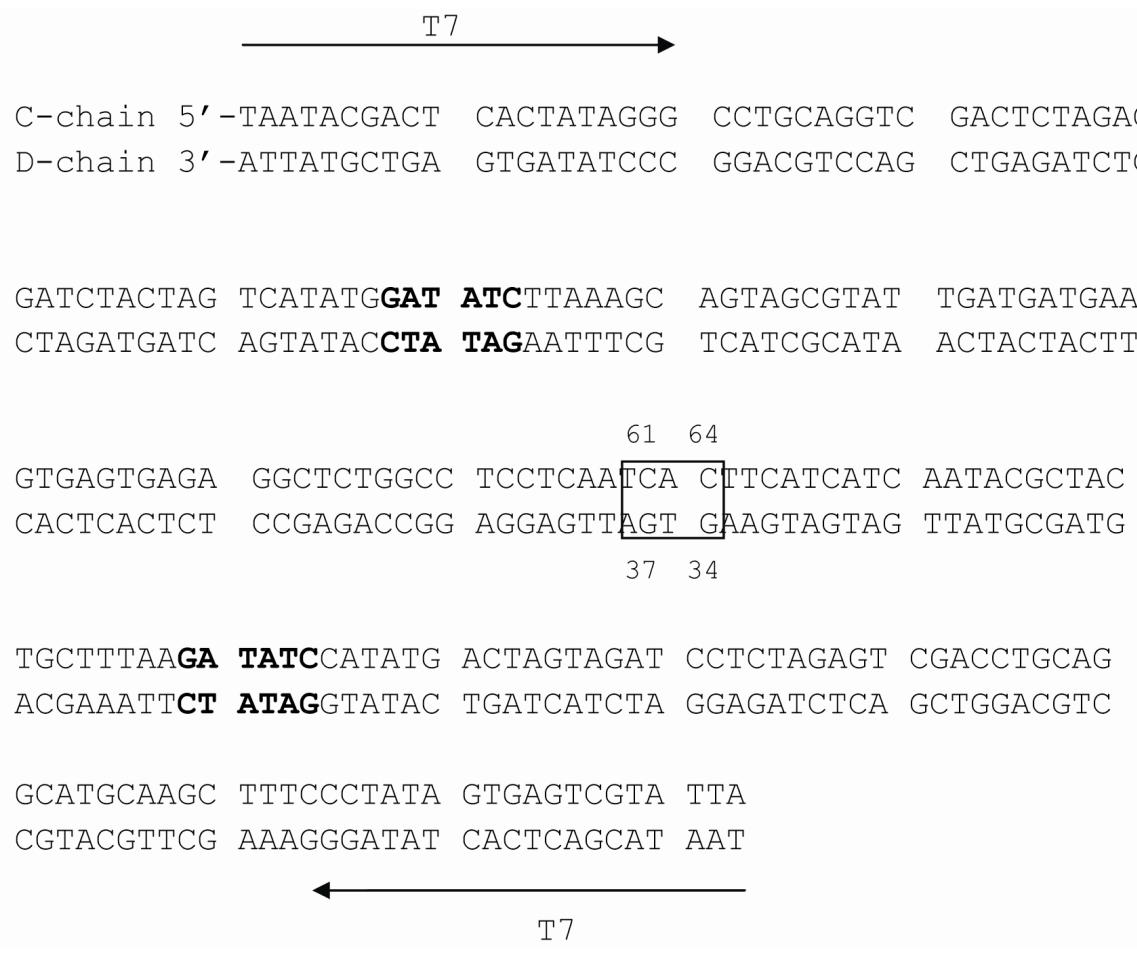

(a)

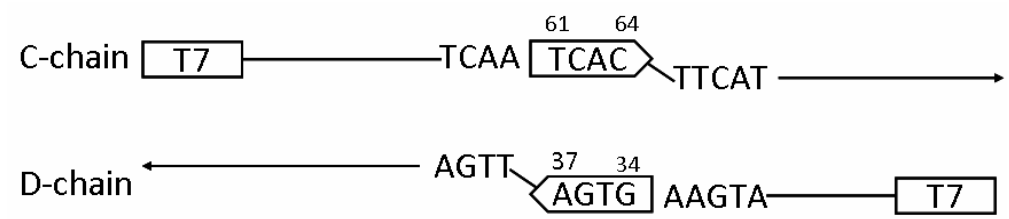

(b)

Figure 7. (a) The partial nucleotide sequence of b6-300 as determined using the primer T7. The sequence used as the primer is indicated by arrows. The 4-bp structures presumably involved in the initial hybridization are boxed. The EcoRV sites are shown in bold face; (b) A possible mechanism for generation of the portion contained in b6-300. C-chain was elongated from $\mathrm{T} 7$ to form the tetranucleotide $-\mathrm{T}^{61}-\mathrm{C}-\mathrm{A}-\mathrm{C}^{64}$ by reading the template and $\mathrm{D}$-chain was elongated from the same primer to form $\mathrm{A}^{37}-\mathrm{G}-\mathrm{T}-\mathrm{G}^{34}-$. After initial hybridization between these tetranucleotides, subsequent elongation was started by reading the sequence of the respective counterchain to complete a double strand structure between $\mathrm{C}$ and D-chains.

nucleotide $\mathrm{C}^{2926(-)}-\mathrm{T}-\mathrm{G}-\mathrm{G}-\mathrm{C}^{2922(-)}$ and the complementary $\mathrm{G}^{3002(-)}-\mathrm{A}-\mathrm{C}-\mathrm{C}-\mathrm{G}^{3006(-)}$ as indicated in Figure 8(c), and subsequent elongation was achieved by reading the sequence of the counter chain.

\section{DISCUSSION}

PCR is useful for amplifying nucleotides having a sequence expected from the use of forward and reverse primers [9]. However, as shown in Figures 3, 7, and 8, PCR gave unexpected results under certain conditions. In the present case, when the two chains were elongated by reading the sequence of the respective template until the completion of a certain hybridizable short (3 - 5) nucleo- tide structure, subsequent elongation was started by reading the sequence of the counter nucleotide chain that had been elongated from the respective template. The short nucleotide structure participating in the initial hybridization was present at multiple positions of the template used. It is possible that elongation of the chain by reading the template was interrupted once before the completion of amplification due to the template's palindrome region which had formed a double strand structure during PCR as exemplified by a model for the process during production of b6-110 (Figure 8(d)). Then, the accumulated short nucleotides were hybridized each other through the complementary nucleotide sequence to start elongation by reading the sequence of the counter chain 


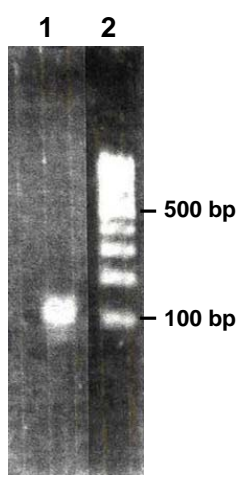

(a)

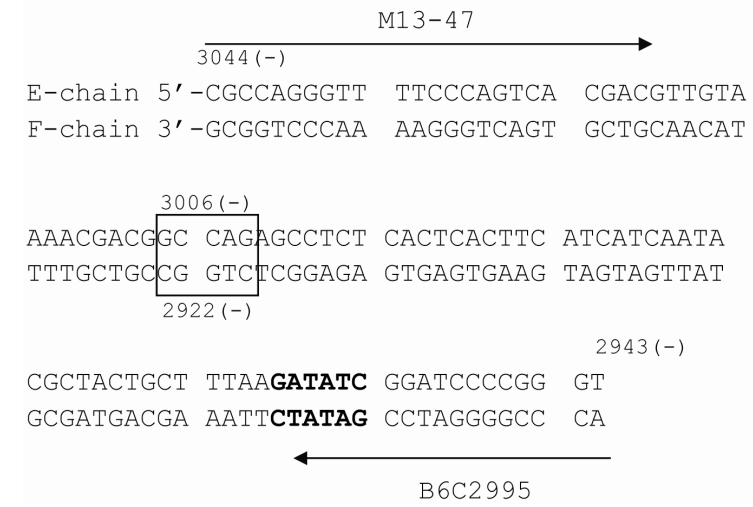

(b)
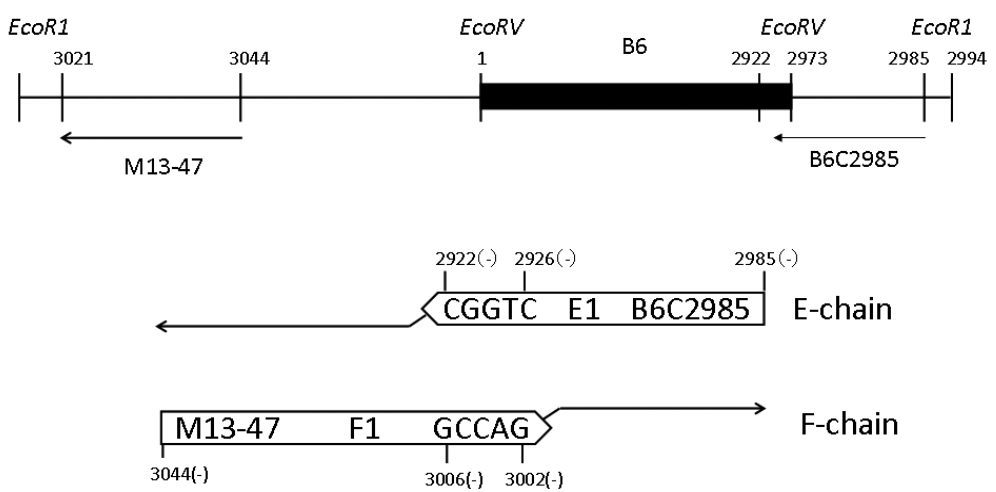

(c)

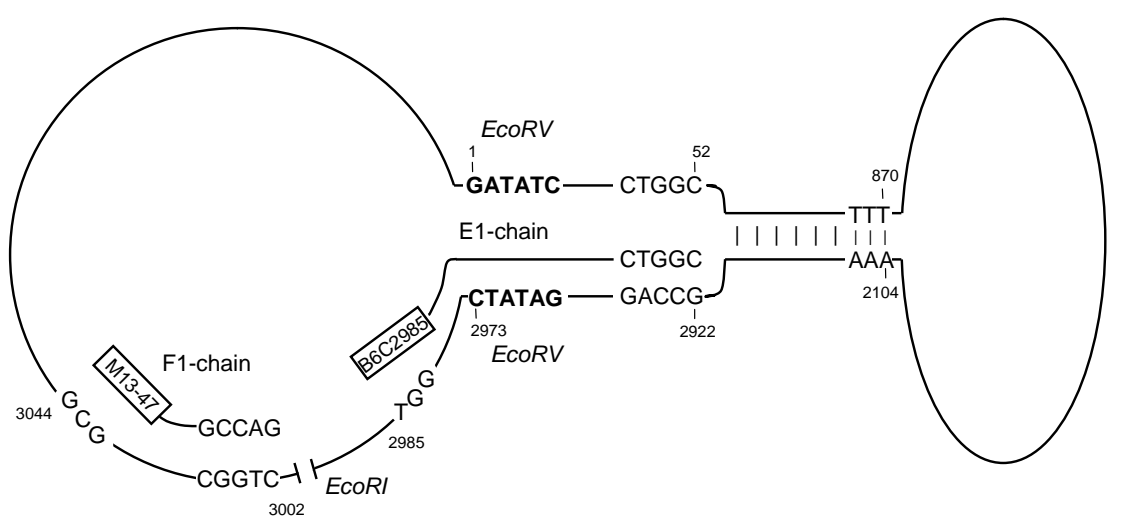

(d)

Figure 8. (a) Agarose gel electrophoresis of a product (b6-110) of PCR using the EcoRI digest of B6 and primers B6C2985 and M13-47 (lane 1) and a size marker (lane 2); (b) Nucleotide seence of b6-110. The sequences used for primers are indicated by arrows. The pentanucleotide structure used presumably for initial hybridization is boxed. The sequences used as primers are indicated by arrows. The EcoRV site is shown in bold face; (c) A presumed mechanism for generation of b6-110. E-chain was produced by elongation from B6C2985 to a -C-T-G-G-C structure, followed by reading of the sequence of the preformed portion of F-chain. F-chain was produced by elongation from primer M13-47 to a -G-C-C-A-G structure, followed by reading of the sequence of the preformed portion of E-chain. E1 and F1 represent portions which were elongated from B6C2985 and M13-47 with subsequent interruption by formation of mutually hybridizable pentanucleotides, respectively. The black bar indicates B6; (d) A proposed mechanism for generation of b6-110 depending on the palindrome structure in B6. B6 contains the sequence derived from bovine genomic DNA $\left(\mathrm{G}^{1}-\mathrm{A}-\mathrm{T}-\mathrm{A}\right.$ ---T-A-T-C ${ }^{2973}$ ) in which the portion of $\mathrm{G}^{1}-\mathrm{A}-\mathrm{T}-\mathrm{A}$-------T-T-T ${ }^{870}$ is able to form base-pairing with a portion of $\mathrm{C}^{2973}$-T-A-T--------A-A-A ${ }^{2104}$. Production of E1-chain was started by binding of the primer B6C2985 to the complementary sequence in the template and interrupted by the formation of a -C-T-G-G-C structure. Production of F1-chain was started by binding of the primer M13-47 to the complementary sequence in the template and interrupted by formation of a -G-C-C-A-G structure. The temporal accumulation of E1- and F1-chains promoted the initial hybridization and subsequent elongation by reading the preformed sequence of the respective counter chain to complete the double strand nucleotide of b6-110 as shown in (c)). 
preformed during PCR.

Such an unusual amplification generated under certain conditions in a DNA sequence may be one of the mechanisms for the genetic recombination found in our previous study [8]. Study to examine possible occurrence of $E$. coli DNA-derived nucleotide sequences in bovine genomic DNA is now in progress.

\section{REFERENCES}

[1] Hagerman, A.E. and Butler, L.G. (1981) The specificity of proanthocyanidin-protein interactions. The Journal of Biological Chemistry, 256, 4494-4497.

[2] Carlén, A., Bratt, P., Stenudd, C., Olsson, J. and Strömberg, N. (1998) Agglutinin and acidic proline-rich protein receptor patterns may modulate bacterial adherence and colonization on tooth surfaces. Journal of Dental Research, 77, 81-90. doi:10.1177/00220345980770011301

[3] Tamaki, N., Tada, T., Morita, M. and Watanabe, T. (2002) Comparison of inhibitory activity on calcium phosphate precipitation by acidic proline-rich proteins, statherin, and histatin-1. Calcified Tissue International, 71, 59-62. doi:10.1007/s00223-001-1084-0

[4] Levine, M. (2011) Susceptibility to dental caries and the salivary proline-rich proteins. International Journal of Dentistry, 2011, Article ID 953412. doi:10.1155/2011/953412

[5] Isemura, S. and Saitoh, E. (1994) Molecular cloning and sequence analysis of cDNA coding for the precursor of the human salivary proline-rich peptide P-B. The Journal of Biochemistry (Tokyo), 115, 1101-1106.

[6] Strawich, E. and Glimcher, M.J. (1990) Tooth "enamelins" identified mainly as serum proteins. Major "enamelin" is albumin. European Journal of Biochemistry, 191, 47-56. doi:10.1111/j.1432-1033.1990.tb19092.x

[7] Isemura, S., Watanabe, S., Fujiwara, S. and Sanada, K. (2004) Tissue distribution and nucleotide sequence of bovine mRNA for salivary proline-rich protein P-B. Archives of Oral Biology, 49, 881-887. doi:10.1016/j.archoralbio.2004.06.001

[8] Sato, R., Isemura, S., Fujiwara, S. and Sanada, K. (2005) Evidence for inclusion of a segment of Escherichia coli genomic DNA in bovine tooth germ mRNA encoding salivary proline-rich protein P-B. Biomedical Research, 26, 153-158. doi:10.2220/biomedres.26.153

[9] Saiki, R.K., Gelfand, D.H., Stoffel, S., Scharf, S.J., Higuchi, R., Horn, G.T., Mullis, K.B. and Erlich, H.A. (1988) Primer-directed enzymatic amplification of DNA with a thermostable DNA polymerase. Science, 239, 487-491. doi:10.1126/science. 2448875 